\title{
Análisis de los municipios chilenos: ingresos por gestión versus transferencias del Fondo Común Municipal
}

\author{
Mario Pérez \\ Servicio de Impuestos Internos, Chile
}

\section{Resumen}

Este estudio se focaliza en analizar los posibles efectos en los ingresos propios por gestión de los municipios si se transfieren más recursos mediante el Fondo Común Municipal (FCM). Para esto se utiliza un modelo de regresión de efectos fijos con datos de panel entre los años 2009 al 2012, obtenidos del Sistema Nacional de Información Municipal (SINIM). Las transferencias provenientes del FCM impactan negativamente en los ingresos por gestión propia solo a un grupo de comunas (47), clasificadas por la Subsecretaría de Desarrollo Regional (SUBDERE) como "Grandes comunas metropolitanas con alto y/o medio desarrollo", siendo en promedio casi 5 unidades menos los ingresos por gestión recaudados por 1 unidad más de aporte del FCM. Sin embargo para el resto de los municipios del país no existe evidencia suficiente para determinar que existe este fenómeno conocido como "pereza fiscal" (Raich, 2004).

Palabras clave: Financiamiento fiscal municipal, descentralización, pereza fiscal, Fondo Común Municipal.

Chilean Municipalities Analysis: Management Revenues versus Transfers from the Municipal Common Fund

\begin{abstract}
This study focuses on analysing the possible effects on the own revenues from municipalities management if more resources are transferred through the Municipal Common Fund (FCM). For this, a fixed-effect regression model with panel data between 2009 and 2012, obtained from the National Municipal Information System (SINIM), is used. Transfers from the FCM have a negative impact on own-managed revenues only to a group of communes (47), classified by the Sub-Secretariat for Regional Development (SUBDERE) as "Big metropolitan communes with high and / or medium development". In these communes, the average is almost 5 units less of revenue per management collected by 1 unit plus contribution of the FCM. However, for the rest of the municipalities of the country, there is insufficient evidence to determine that this phenomenon known as "fiscal laziness" exists (Raich, 2004).
\end{abstract}

Keywords: Municipal fiscal financing, decentralization, fiscal laziness, Municipal Common Fund.

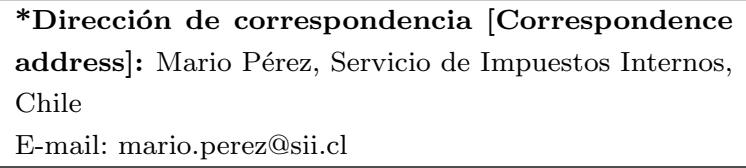




\section{Introducción}

La descentralización, según CEPAL (1993), se puede definir como un proceso de trasferencias de competencias y recursos desde la administración central de un determinado Estado hacia las administraciones sub nacionales. De acuerdo a Galilea Ocón et al. (2011), la descentralización presenta dos dimensiones, la "institucional" que se expresa en las normas que definen el ámbito de las responsabilidades de cada nivel de gobierno, conceden espacio de libertades y/o restringen el rango de acción y definen las fuentes de financiamiento para su ejecución, la segunda dimensión es la "descentralización fiscal" ligada a la primera y se centra en la magnitud relativa de los recursos disponibles a nivel descentralizado y/o el volumen de gastos de los gobiernos sub nacionales en relación al gasto del gobierno general.

En Chile, la descentralización fiscal a nivel de municipios es reducida en relación a los países desarrollados. De acuerdo a lo recopilado por Horst (2009), el gasto municipal respecto al gasto del gobierno central es del orden del 12,5\%, mientras que en los países unitarios de la OCDE dicha tasa alcanza el $30 \%$ aproximadamente.

Existe literatura que muestra que los conceptos "descentralización" y "desigualdad" se encuentran muy relacionados entre sí. Por ejemplo, Prats (2009) señala abiertamente que si no se resuelve la excesiva concentración económica, política y territorial del poder en Chile no se logrará el desarrollo esperado. Asimismo, Rosales (2012) afirma que los temas de pobreza e inequidad social son más cercanos desde lo local que desde otras esferas y, por tanto, desde ahí se pueden abordar mejor dichos problemas. Por su parte, según la OCDE (2009), Chile presenta un alto grado de concentración económica y que es indispensable impulsar políticas de descentralización.

\section{El rol de los municipios en Chile}

En Chile la ley № 18.695 , Orgánica Constitucional de Municipalidades, dictamina que la administración local de las comunas reside en las municipalidades. De acuerdo al artículo $1^{\circ}$ de esta ley, las municipalidades son corporaciones autónomas de derecho público, con personalidad jurídica y patrimonio propio, cuya finalidad es satisfacer las necesidades de la comunidad local y asegurar su participación en el progreso económico, social y cultural de las respectivas comunas, asimismo, el artículo $2^{\circ}$ declara que las municipalidades estarán constituidas por el alcalde, que será su máxima autoridad, y por el concejo. CEPAL (2005) destaca una serie de modificaciones producto de la reforma de 1999 a esta ley, principalmente las siguientes:

- Se amplió la potestad a la municipalidad en áreas como la planificación y regulación urbana

- El traspaso de nuevas funciones al municipio debe ir acompañado de los recursos financieros respectivos.

- A los municipios se les otorgó la potestad para el asociativismo municipal y así poder optimizar los recursos para hacer frente a problemas comunes.

- La obligatoriedad de coordinar los servicios públicos en el territorio comunal y

- Mayor capacidad de fiscalización al concejo municipal.

Sin embargo, este trabajo de CEPAL (2005) es crítico al señalar que si bien se han consolidado logros en el fortalecimiento de las competencias a nivel municipal, todavía el proceso de descentralización está en una fase de maduración, debiendo ser revisado y adecuado para garantizar el cumplimiento del mandato legal: satisfacer las necesidades de la comunidad. Lo anterior se debería esencialmente a la insuficiencia de personal calificado en la gran mayoría de los municipios, sobre todo en las comunas más rezagadas económicamente por falta de recursos, impidiendo avanzar en la superación de la brecha territorial del desarrollo del país.

\section{Financiamiento de los municipios en Chile}

Como recopilaron Henríquez et al. (2011), las principales fuentes de ingreso de una administración local son:

1. Las transferencias recibidas desde el resto del sector público $\mathrm{y}$

2. Los ingresos propios municipales.

Las primeras, son recursos provenientes del gobierno central para financiar directamente programas sectoriales y políticas públicas, tales como educación pública y salud primaria, además de vivienda, protección social, seguridad, urbanismo y otros proyectos de inversión (obtenidos a través del Fondo Nacional de Desarrollo Regional). Los segundos, se componen de impuestos, patentes, derechos 
y permisos para actividades empresariales, uso de bienes públicos y entrega de servicios municipales.

En lo que respecta a impuestos se encuentra el impuesto territorial, conocido también como contribuciones, que es administrado por el gobierno central, ya que el cobro y fiscalización es gestión del Servicio de Impuestos Internos (SII) y cuya recaudación se encuentra a cargo la Tesorería General de la República (TGR), también las exenciones a este tributo son definidas por ley. En el caso de las patentes comerciales, los municipios pueden fijar una tasa dentro de un rango fijado por ley, dependiendo del capital propio de las personas y/o empresas declarado, pero su recaudación depende exclusivamente de los municipios y no de la TGR. En cuanto a permisos de circulación, es el Servicio de Impuestos Internos (SII) quien define el avalúo fiscal de los vehículos y las tasas están fijadas también por ley, pero dicho permiso se puede obtener en cualquier municipalidad del país, asimismo, la recaudación también depende exclusivamente de cada municipio.

Donde existe mayor grado de autonomía es en los derechos municipales, siendo el derecho de extracción de residuos domiciliarios el más importante (Bravo, 2011a), en el cual cada municipio fija una tarifa de acuerdo a sus costos, la cual puede ser modificada vía ordenanza si es necesario antes que termine el plazo (por ley la tarifa debe regir por tres años). Además, los criterios de rebaja y/o exenciones quedan a criterio de los municipios aunque por ley están exentas las viviendas cuyo avalúo fiscal sea menor a 225 Unidades Tributarias Mensuales (UTM).

Parte de estas fuentes constituyen los recursos del Fondo Común Municipal (FCM), que luego son redistribuidos a los municipios. El FCM está definido por la Constitución Política de la República (Artículo 122) como un "mecanismo de redistribución solidaria de los ingresos propios entre las municipalidades del país".

La configuración actual del FCM y sus mecanismos de funcionamiento han sido determinados principalmente a través de la Ley № 20.237 , promulgada el año 2007, la cual modificó el D.L. № 3.063, de 1979, sobre Rentas Municipales; la Ley № 18.695 , Orgánica Constitucional de Municipalidades; y el Decreto № 1293 del Ministerio del Interior, promulgado el año 2009, que establece aspectos de procedimiento y fórmulas de cálculo de los coeficientes utilizados. Es importante señalar que a partir de la Ley $\mathrm{N}^{\circ} 20.033$ del año 2005 se pudo establecer un monto fijo de aporte fiscal (gobierno central) para este Fondo (218.000 UTM), ya que anteriormente dependía de la ley de presupuesto el monto desti- nado para este fin.

La estructura actual (operativa desde el 2008 hasta ahora) para distribuir el FCM entre las municipalidades es la siguiente ${ }^{1}$ :

- $25 \%$ partes iguales

- $35 \%$ si tienen menos Ingresos Propios Permanentes (IPP) respecto al año anterior, en relación con el promedio nacional de dicho ingreso por habitante.

- $10 \%$ de acuerdo al número de pobres de la comuna, ponderado en relación con la población pobre del país.

- $30 \%$ en proporción directa al número de predios exentos de impuesto territorial de cada comuna, con respecto al número de predios exentos del país, ponderado según el número de predios exentos de la comuna en relación con el total de predios de ésta.

Por tanto, en teoría, un $60 \%$ de este Fondo (A y B) no generaría incentivos para mejorar la gestión de ingresos propios de los municipios, lo anterior ha sido expuesto por distintos autores (Horst, 2009; Ormeño, 2010; Bernstein e Inostroza, 2009). Mientras que solo un 40 \% (C y D) son componentes que están relacionados directamente con el objetivo principal de este sistema, la redistribución solidaria entre los municipios.

\section{Análisis del Fondo Común Municipal}

Según los datos obtenidos del Sistema Nacional de Información Municipal (SINIM), dependiente de la SUBDERE se observa que el FCM cumple su fin redistributivo. A continuación se describe que ocurre al agrupar las municipalidades en quintiles según el Ingreso Propio Permanente (IPP) per cápita y el porcentaje del FCM asignado para cada uno $^{2}$.

De acuerdo a la figura 1 el quintil de menores ingresos propios (quintil 1) posee una mayor proporción del FCM que el quintil de mayores ingresos propios (quintil 5) lo cual cumple con el objetivo de este Fondo; sin embargo, al igual que lo recopilado

\footnotetext{
${ }^{1}$ También existe un mecanismo de estabilización, si los ingresos por FCM son menores que los estimados para el año, esta diferencia podrá ser compensada total o parcialmente, dependiendo si el monto total de las reducciones es menor o mayor que el monto total de los incrementos de las comunas con recursos adicionales

${ }^{2}$ Metodología usada por Henríquez et al. (2011), aunque en dicho estudio se analizó entre los años 2001-2009
} 
por Henríquez y otros (2011), los quintiles 2, 3 y 4 no responden a dicha lógica de distribución.

Por su parte, si a las comunas de estos mismos quintiles se les suma el FCM per cápita para estimar cuál es el "ingreso total per cápita", resulta que tanto el promedio (figura 2) como la mediana (figura 3) muestran una tendencia donde el quintil 1 tiene mayores "ingresos totales per cápita" que los quintiles 2 y 3 , mientras que el quintil 4 posee mayores ingresos que sus antecesores, quedando finalmente el quintil 5 con una diferencia muy superior al resto en ambos casos.

Es necesario señalar que un componente que genera reparos al funcionamiento del FCM es su "baja efectividad neta" como plantea Valenzuela (2008) en un trabajo desarrollado para el Banco Interamericano del Desarrollo, ya que un parte importante de los recursos recaudados regresan a los mismos municipios aportantes, mientras que la mayor parte de los recursos netos distribuidos son financiados por muy pocos municipios, complejizando con esto la operatoria del FCM ya que en la mecánica de que "todos aportan" y "todos reciben" genera ineficiencias en cuanto a la administración del flujo de dinero.

También se debe considerar que la Ley № 20.237 estableció un mecanismo de estabilización que resulta ser un procedimiento destinado a compensar a las municipalidades que, por aplicación de las normas de distribución del FCM, vean reducidos sus ingresos estimados a percibir durante el año de distribución, en relación al año del cálculo, y que permite la compensación total o parcial de las diferencias que se produzcan. Esto último generaría efectos adversos como plantea Bravo (2011b), aunque lamentablemente según esta autora no se ha medido el impacto de esta modificación a la distribución del FCM.

\section{Definición de "esfuerzo fiscal" y "pe- reza fiscal"}

De acuerdo con López y Castellanos (2002), el "esfuerzo fiscal" se define como la relación entre la recaudación efectiva de un territorio respecto al potencial recaudatorio de la área administrativa correspondiente. Según Raich (2004), en la literatura de finanzas públicas locales hay dos explicaciones alternativas sobre los impactos de las transferencias en el "esfuerzo fiscal", una de ellas conocida como "pereza fiscal" que sostiene que el incremento de las transferencias ocasiona una disminución de dicho esfuerzo por parte de los gobiernos locales que reciben las transferencias, ya que los que se "esfuerzan lo menos, obtienen lo más". Este autor señala que esto se debe a que los gobiernos actúan de manera racional y simplemente prefieren recibir más transferencias que hacer frente a los altos costos políticos y administrativos de la recaudación tributaria. No obstante, Bravo (2011a) afirma que en países como Chile, donde las tasas impositivas y la definición de las bases son determinadas por el gobierno central y a su vez los municipios son los responsables de recaudar aquellos impuestos ${ }^{3}$, trayendo consigo un reducido campo de acción para los municipios ${ }^{4}$ y por lo tanto una dificultad para medir "esfuerzo fiscal", la literatura empírica se ha focalizado en estudiar los efectos de las transferencias en los ingresos locales, usando tales ingresos como proxy de "esfuerzo fiscal", ya que no existe una interacción estratégica entre los distintos gobiernos locales para recaudarlos, si existiera dicha interacción todas las municipalidades podrían aumentar $\mathrm{su}$ "esfuerzo fiscal" sin incrementar necesariamente su recaudación.

\section{Algunos estudios empíricos sobre "pereza fiscal" en gobiernos locales de América Lati- na}

Existen investigaciones sobre "pereza fiscal" en países latinoamericanos, las cuales han usado metodologías distintas y con resultados diversos, por ejemplo Raich (2004) analizó 217 municipios del Estado de Puebla, México, respecto a la recaudación de ingresos propios antes y después de la creación del Fondo de Aportaciones para la Infraestructura Social Municipal (FAISM), encontrando que dicho fondo afectó adversamente el esfuerzo fiscal de tales municipios, sin embargo la magnitud de este impacto difiere según población y niveles de bienestar, siendo mayor en los municipios pocos poblados y con niveles de bienestar bajos. Por su parte Cadena (2002), mediante la metodología de diferencias en diferencias, consideró 203 municipios colombianos distribuidos en todo el país y sus conclusiones fueron que el comportamiento de los ingresos tributarios municipales mejoró simultáneamente con el aumento de transferencias, especialmente para el caso del impuesto predial, siendo los municipios pequeños y con mayor ruralidad los que presentaron mayor esfuerzo fiscal, descartando así la existencia de "pereza fiscal", al igual que trabajos anteriores realizados en dicho país. Asimismo, Herrera y Francke (2009) tomaron 1686 municipalidades del Perú, midiendo el nivel de eficiencia del gasto municipal y los factores que la determinarían, encontran-

\footnotetext{
${ }^{3}$ La ley chilena define como impuestos municipales las patentes comerciales y el permiso de circulación

${ }^{4}$ En el caso de Chile se hace referencia a su escasa capacidad recaudatoria en el apartado Financiamiento de los municipios en Chile.
} 
Figura 1: Composición por quintil de IPP per cápita de los ingresos por FCM, años 2008-2011

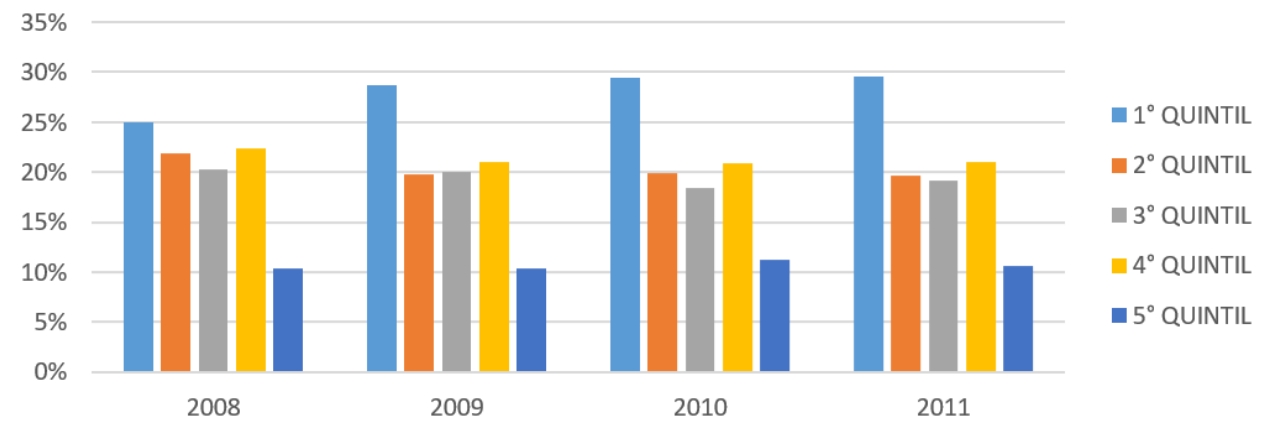

Fuente: Elaboración propia basada en SINIM.

Figura 2: Promedio de la suma IPP per cápita y FCM per cápita en miles de pesos nominales

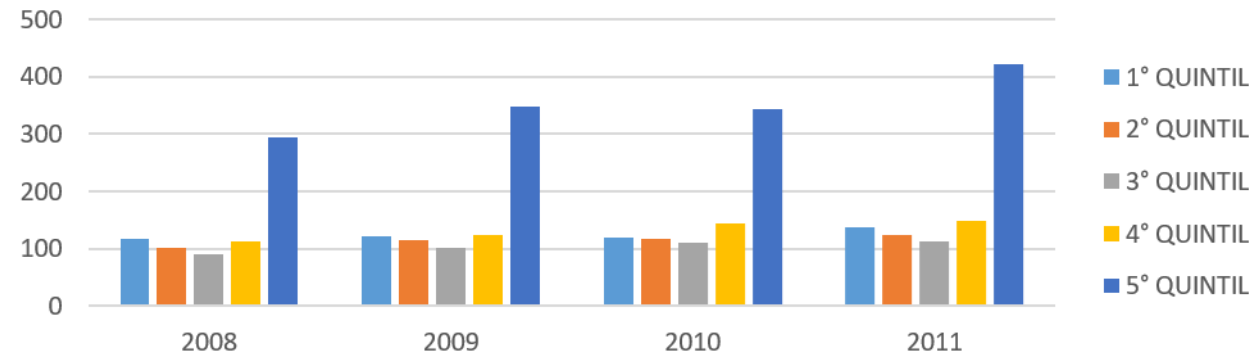

Fuente: Elaboración propia basada en SINIM.

Figura 3: Mediana de la suma IPP per cápita y FCM per cápita en miles de pesos nominales

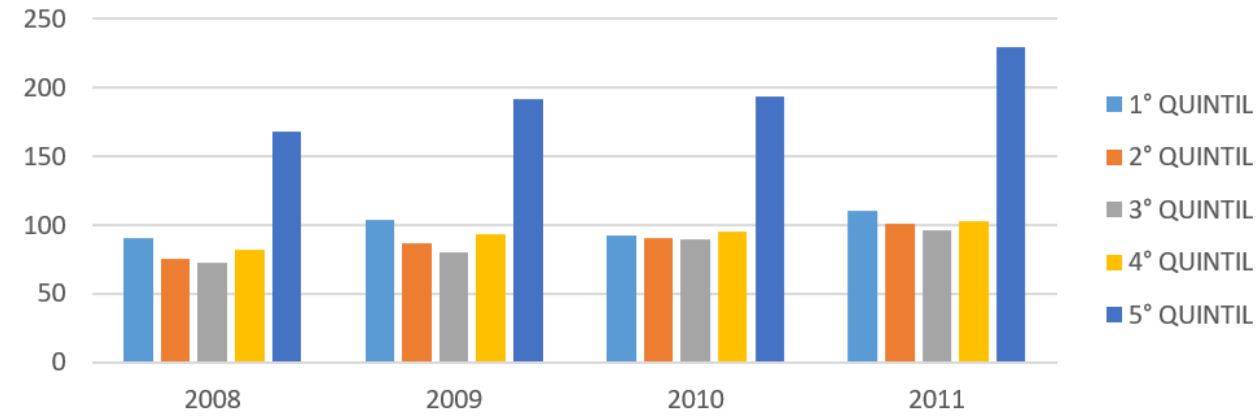

Fuente: Elaboración propia basada en SINIM. 
do que las transferencias por el Fondo de Compensación Municipal (FONCOMUN), en general, tuvieron una incidencia negativa en la eficiencia de las municipalidades, aunque con algunas excepciones en municipalidades clasificadas como urbanas y metropolitanas. Esta breve recopilación de investigaciones da cuenta lo expuesto por Bird y Slack (1993) que no es factible llegar a un acuerdo sobre la magnitud del impacto de las transferencias en el esfuerzo fiscal.

\section{Estudios nacionales respecto al Fondo Común Municipal y "pereza fiscal"}

Según Henríquez et al. (2011), la literatura nacional que refiere al FCM con posterioridad a la reforma llevada a cabo en 2007, no atiende en específico el comportamiento de dicho instrumento, sino que aborda tópicos más generales, tales como modernización del Estado, descentralización, autonomía o gestión local (municipal). Aquellos estudios (Horst, 2009; Bernstein e Inostroza, 2009), no hacen referencias en términos cuantitativos de la existencia de "pereza fiscal", aunque plantean que el diseño actual del FCM genera desincentivos para mejorar la gestión de los ingresos propios. Lo anterior es refrendado por Bravo (2011a) señalando que existe muy poca evidencia empírica para el caso chileno, citando solo a Rodríguez (1998), quien encontró una relación inversa entre la recaudación de ingresos locales y las transferencias del FCM recibidas 2 años antes, dicho resultado es válido en el $50 \%$ de los municipios que recibieron mayores recursos provenientes del FCM, aunque no tomó en cuenta el problema de endogeneidad que existe entre dichas transferencias y la recaudación de ingresos (se otorgan más fondos por menos ingresos) sin tener certeza la dirección de causalidad.

Por su parte Bravo (2011a) analizó a 340 municipios chilenos entre los años 1990 y 2007, encontrando evidencia de la existencia de "pereza fiscal", específicamente encontró que un aumento en una desviación estándar del FCM per cápita se asocia con una disminución entre 0,25 y 0,35 desviaciones estándar de ingresos propios per cápita; y al contrario del caso de Rodríguez (1998), utilizó variables instrumentales para controlar el problema de endogeneidad. En dicho estudio, la autora recalca que el efecto neto del incremento de la transferencia es positivo, ya que la reducción de la recaudación de ingresos locales es menor que el incremento en transferencia, alcanzando así el efecto redistributivo deseado del Fondo.

De acuerdo a los antecedentes presentados, la investigación presentada tiene como objetivo el determinar si existe una situación de "pereza fiscal" en los municipios chilenos. Para esto, se utiliza como proxy de "esfuerzo fiscal", los ingresos catalogados como "ingresos por gestión" por parte de SUBDERE. De esta manera, se busca, asimismo, la formulación de lineamientos de políticas para el sistema del FCM, en base a los resultados obtenidos.

\section{Metodología}

Primero se utiliza la clasificación de la SUBDERE utilizada en el Reglamento del Fondo de Incentivo al mejoramiento de la Gestión Municipal (Resolución $\mathrm{N}^{\circ} 115$ del año 2012) para realizar comparaciones con unidades relativamente homogéneas, así los resultados serán interpretables (Herrera y Francke, 2009). Usando distintas variables socioeconómicas y demográficas SUBDERE clasifica a los municipios de Chile en 5 grupos $^{5}$

- Grupo 1: Grandes comunas metropolitanas con alto y/o medio desarrollo (47 comunas) ${ }^{6}$

- Grupo 2: Comunas mayores con desarrollo medio (37 comunas)

- Grupo 3: Comunas urbanas medianas, con desarrollo medio (56 comunas)

- Grupo 4: Comunas semi urbanas y rurales con desarrollo medio (96 comunas)

- Grupo 5: Comunas semi urbanas y rurales con desarrollo bajo (109 comunas)

Además, se emplea un modelo de regresión de efectos fijos con datos de panel desde el año 2009 al 2012, ocupando el ingreso por gestión per cápita (igmpc) ${ }^{7}$ como variable dependiente, este ingreso por gestión es definido en el reglamento antes citado como la sumatoria de las siguientes partidas de ingresos municipales ${ }^{8}$ :

- Patentes municipales (115.03.01.001.000.000)

- Derechos de aseo en patentes municipales (115.03.01.002.002.000)

- Derechos de aseo de cobro directo (115.03.01.002.003.000)

\footnotetext{
${ }^{5}$ Las variables se agruparon en los siguientes factores: Tamaño, Dispersión, Jerarquía Político Administrativa, Tipo de Localidad, Patrimonio Comunal, Capital Humano, Características Socioeconómicas.

${ }^{6} 34$ de estas comunas pertenecen a la Región Metropolitana.

${ }^{7}$ La población usada en el cálculo per cápita es la que se obtiene de SINIM para cada año.

${ }^{8} \mathrm{Se}$ usa el clasificador presupuestario vigente desde el 2008, ver www.sinim.cl
} 
- Otros derechos (115.03.01.003.000.000)

- Derechos de

(115.03.01.004.000.000)

explotación

- Otras (115.03.01.999.000.000)

- Permisos y Licencias (115.03.02.000.000.000)

- Arriendo de activos no financieros (115.06.01.000.000.000)

- Ingresos de operación (115.07.00.001.000.000)

- Recuperaciones y reembolsos por licencias médicas (115.08.01.000.000.000)

- Multas y sanciones pecuniarias (115.08.02.000.000.000)

Mientras que las variables explicativas del modelo, son las siguientes ${ }^{9}$ :

- FCM per cápita (fcmpc)

- Número de funcionarios de planta cada 1000 habitantes (planta)

- Número de funcionarios de contrata cada 1000 habitantes (contra)

- Si el Plan de Desarrollo Comunal (PLADECO) se encuentra vigente (plan)

- Años en el cargo de alcalde (cargo)

- Si hay elecciones (valor 1 si en dicho año hubo elecciones de alcalde) (eleccion)

- Porcentaje población rural de la comuna (rural)

- Densidad de la población (habitantes/Km2) (denspob)

- Terremoto del año 2010 (terremoto)

Quedando el modelo de la siguiente manera:

$$
\begin{array}{r}
\text { igmpc }=\beta_{0}+\beta_{1} f c m p c+\beta_{2} \text { planta }+\beta_{3} \text { contra }+ \\
\beta_{4} \text { plan }+\beta_{5} \text { cargo }+\beta_{6} \text { eleccion }+\beta_{7} \text { rural }+ \\
\beta_{8} \text { denspob }+\beta_{9} \text { terremoto }+\epsilon
\end{array}
$$

Como fue señalado por Bravo (2011a), uno de los problemas que existe en el desarrollo de trabajos empíricos sobre "pereza fiscal" tiene que ver con

\footnotetext{
${ }^{9}$ Algunas de estas variables recogen lo recopilado por Martínez-Costa y Ordeig (2010) respecto a los factores determinantes de la gestión de las administraciones públicas locales
}

el problema de endogeneidad que existe entre los fondos externos y los ingresos locales, ya que existe dependencia entre ambos (se otorgan más fondos por menos ingresos) sin tener certeza la dirección de causalidad, por lo cual en este trabajo se utiliza una variable instrumental para solucionar aquello. Dicha variable instrumental es el porcentaje de predios exentos de impuesto territorial (prdexen), ya que tiene directa relación en cómo se distribuye el FCM por ley, siendo a su vez una variable exógena a la administración municipal, debido a que las exenciones (montos y condiciones) son definidas por ley en cada reavalúo ${ }^{10}$. Igualmente, la fiscalización y administración de este impuesto corresponde al SII, por lo cual los municipios no pueden modificar directamente mediante su gestión aquella variable, al menos no en el corto y mediano plazo.

La principal fuente de información es el Sistema Nacional de Información Municipal (SINIM) como el Servicio Electoral (SERVEL) para el caso de los años en el cargo del alcalde.

Finalmente, es necesario agregar que las comunas de Las Condes, Vitacura, Providencia y Santiago fueron excluidas del análisis por ser las únicas del país que integran parte de sus ingresos obtenidos por patentes municipales al FCM (65\% las tres primeras y $55 \%$ la última), pudiendo alterar con esto los resultados.

\section{Resultados}

En la tabla 2 se presentan los resultados obtenidos, incorporando previamente al modelo la variable instrumental prdexen para cada uno de los grupos comunales.

La variable instrumental prdexen resultó ser significativa en el grupo 1 al $5 \%$, en el grupo 2 al $1 \%$ y en el grupo 5 al $5 \%$.

De acuerdo a estas cifras, habría respaldo al modelo teórico sobre "pereza fiscal", ya que aparentemente las transferencias provenientes del FCM impactan negativamente en los ingresos por gestión de los municipios, aunque solo al grupo 1 "Grandes comunas metropolitanas con alto y/o medio desarrollo", siendo en promedio casi 5 veces menos lo recaudado por 1 unidad más de aporte del FCM, mientras que para el resto de los municipios no existe evidencia suficiente para determinar que existe dicho problema, incluso en el grupo 5 "Comunas semi urbanas y rurales con desarrollo bajo" pudiera haber un efecto positivo producto del aporte del FCM, sin embargo es necesario señalar que en este

\footnotetext{
${ }^{10}$ Ley $\mathrm{N}^{\circ} 17.235$ sobre Impuesto Territorial.
} 
Tabla 1: Estimaciones obtenidas del modelo para cada grupo comunal

\begin{tabular}{lccccc}
\hline & Grupo 1 & Grupo 2 & Grupo 3 & Grupo 4 & Grupo 5 \\
\hline fcmpc & $-5,24^{* *}$ & $-1,01$ & $-0,50$ & 0,03 & $3,03^{* *}$ \\
& $(2,14)$ & $(0,78)$ & $(0,86)$ & $(1,46)$ & $(1,26)$ \\
planta & 0,69 & $-22,62^{*}$ & 2,87 & $-4,62$ & $-29,24^{* *}$ \\
& $(2,04)$ & $(7,15)$ & $(4,74)$ & $(10,61)$ & $(11,68)$ \\
contra & 5,60 & 0,97 & 3,86 & 6,50 & $-35,08$ \\
& $(7,91)$ & $(3,00)$ & $(4,74)$ & $(4,49)$ & $(24,69)$ \\
plan & $-1,40$ & 7,38 & $-4,00$ & 3,37 & $-37,23$ \\
& $(11,23)$ & $(4,88)$ & $(4,19)$ & $(21,47)$ & $(51,56)$ \\
cargo & $12,10^{*}$ & $4,34^{* *}$ & 5,10 & 7,04 & $-22,35$ \\
& $(2,88)$ & $(1,77)$ & $(3,58)$ & $(9,31)$ & $(16,62)$ \\
eleccion & $8,49^{* *}$ & $4,45^{* * *}$ & 1,37 & $-1,74$ & $-13,13$ \\
& $(4,11)$ & $(2,35)$ & $(2,79)$ & $(8,87)$ & $(22,96)$ \\
rural & 2,09 & $-1,30$ & $-1,47$ & $-1,63$ & $-2,75$ \\
& $(15,02)$ & $(1,24)$ & $(2,45)$ & $(1,49)$ & $(7,14)$ \\
denspob & $-0,01^{* * *}$ & $-0,09$ & $-0,27$ & $-0,24$ & 30,70 \\
& $(0,01)$ & $(0,08)$ & $(0,23)$ & $(1,99)$ & $(25,64)$ \\
terremoto & $-10,41^{*}$ & $-4,47^{* *}$ & $-2,41$ & 1,07 & $37,98^{* * *}$ \\
& $(3,91)$ & $(2,04)$ & $(2,69)$ & $(8,93)$ & $(22,29)$ \\
\hline Núm. obs. & 167 & 143 & 210 & 348 & 388 \\
$R^{2}$ & 0,23 & 0,46 & 0,40 & 0,14 & - \\
\hline$* * * p<0,01,{ }^{* *} p<0,05,{ }^{*} p<0,10$ & & &
\end{tabular}

grupo existe muy poca variación de acuerdo a las variables usadas en el modelo, por lo cual la estimación no arroja un valor para el $\mathrm{R} 2$, impidiendo hacer conclusiones más sustantivas al respecto.

Analizando las otras variables que pudieran afectar la gestión de ingresos propios, los años en el cargo del alcalde (cargo), como si es un año de elecciones municipales (elección) afectarían positivamente a los municipios de las comunas más desarrolladas (grupos 1 y 2), esto podría indicar que la experiencia de ejercer la función de alcalde, quien es finalmente el responsable de gestionar el municipio, ayudaría en efecto a mejorar la recaudación y/o buscar nuevas fuentes de ingresos, inclusive, en año de elecciones los ingresos por gestión aumentaría, quizás para solventar un mayor gasto efectuado en el período ${ }^{11}$.

Por su parte, a los grupos 1 y 2 el factor terremoto del año 2010 pudiera haber afectado de manera negativa los ingresos por gestión propia de estos municipios, sin embargo, sucedería lo contrario en el caso del grupo 5. Lo anterior se podría explicar porque producto del terremoto se realizaron diversas campañas en ayuda de las comunas más afectadas y con menores recursos, incluyendo, por ejemplo, que los permisos de circulación fuesen obtenidos en tales municipios para mejorar así sus finanzas ${ }^{12}$, de

\footnotetext{
${ }^{11}$ Se debe precisar que esta afirmación no ha sido cotejada con los datos respectivos al gasto municipal.

${ }^{12}$ Esta información fue obtenida de una entrevista en el mes de junio del año 2013 con José Palominos Pino, funcio-
}

todos modos, como fue señalado anteriormente, las estimaciones para este grupo presentan un problema estadístico por lo cual sus resultados serían solo referenciales.

Para el grupo 1, la variable densidad poblacional (denspob) tendría un impacto negativo, esto podría deberse a que son comunas que tienen una gran concentración de habitantes, por tanto, al aumentar este factor pudiera superar la capacidad de gestión existente en los municipios.

En el caso de la variable funcionarios de planta por cada 1000 habitantes (planta), tanto en el grupo 2 como en el grupo 5 (con los resguardos pertinentes en la interpretación para este grupo), una mayor cantidad de funcionarios generarían una menor cantidad de ingresos por gestión, siendo una posible explicación lo planteado por Horst (2009), en cuanto a que la planta de funcionarios se encuentra fijada por ley y que en la práctica el estatuto administrativo impide la flexibilidad necesaria para que la autoridad edilicia gestione eficientemente el recurso humano, pudiendo ser estos resultados señal de sobre dotación del personal. Sin embargo, esta situación ocurriría en solo 2 grupos de comunas, "Comunas mayores con desarrollo medio" y "Comunas semi urbanas y rurales con desarrollo bajo", por lo cual no sería posible señalar que efectivamente la rigidez del estatuto administrati-

nario de SUBDERE del Departamento de Finanzas Municipales. 
vo sea una problemática a nivel nacional y quizás pueda deberse a otras causales tal disminución, por ejemplo, falta de capacitación del personal.

Considerando además los resultados obtenidos por Bravo (2011a) ${ }^{13}$, habría evidencia empírica que en Chile existe "pereza fiscal" en las administraciones locales, sin embargo, este problema al parecer se concentraría solo en un grupo reducido de comunas, las "Grandes comunas metropolitanas con alto y/o medio desarrollo", y no necesariamente en todo el país, por tanto, según los resultados de este estudio, un aumento de transferencias provenientes del FCM no implicaría necesariamente que los ingresos por gestión de los municipios se vean disminuidos, incluso, pudiera ser que las comunas más rezagadas (grupo 5) a partir de este aumento de transferencias puedan obtener mayores ingresos propios, similar a lo reportado por Cadena (2002) en el caso colombiano.

\section{Conclusiones y recomendacio- nes}

Primero, los resultados obtenidos en este trabajo van en línea con la política de aumentar las transferencias vía FCM, ya que no existiría evidencia que dicho aumento perjudique la generación de ingresos por gestión en la gran mayoría de los municipios y además este Fondo sí cumpliría con el objetivo de redistribuir de manera solidaria a las comunas con menos recursos. Asimismo, en el análisis del FCM del capítulo introductorio, como las estimaciones según el modelo propuesto en esta investigación, se obtiene cierta evidencia a favor de modificar el actual mecanismo de distribución del FCM, pudiendo ser una alternativa diseñar el mecanismo de tal forma que las comunas con mejor dotación de recursos (especialmente las del grupo 1) perciban menos transferencias por esta vía, en términos concretos, el ítem del $25 \%$ del FCM que se reparte actualmente en partes iguales se pudiera distribuir mediante una ponderación distinta dependiendo a que grupo pertenece cada comuna.

Segundo, bajo el actual sistema en que opera el FCM, para aumentar los recursos existen dos alternativas, no excluyentes entre sí:

- Mayor aporte del gobierno central

- Mayor aporte por parte de las comunas con mayores ingresos.

\footnotetext{
${ }^{13}$ En el trabajo de Bravo se utilizaron datos desde 1990 hasta el año 2007
}

Como referencia para la primera alternativa, si los ingresos totales del FCM distribuidos el año 2012, según los datos de SINIM $^{14}$, alcanzaron los $\$ 784.125 .366 .000$ pesos y el aporte de 218.000 UTM en dicho año alcanzó los $\$ 8.396 .924 .000$ pesos $^{15}$, el aporte fiscal en términos relativos alcanzó solo a $1,1 \%$ aprox., aunque en este cálculo no se considera el pago de impuesto territorial de los bienes raíces fiscales esta cifra no debiera cambiar considerablemente, por lo cual, este monto pudiera ser aún un porcentaje insuficiente.

Respecto a la segunda opción, se sugiere que estos mayores aportes provengan principalmente de los ingresos del impuesto territorial, ya que son ingresos que no se ven influenciados directamente por la gestión de los municipios, evitando así posibles "incentivos perversos" para recaudar menos de lo óptimo. En la práctica, sería conveniente incorporar una o más comunas pertenecientes al grupo 1 para que aporten un $65 \%$ del impuesto territorial, al igual que las comunas de Las Condes, Vitacura, Providencia y Santiago, siendo un claro ejemplo la comuna de Lo Barnechea, la cual presentó en el año 2012 un superávit presupuestario sobre los $14 \mathrm{mil}$ millones de $\operatorname{pesos}^{16}$. Si se hubiese modificado dicho porcentaje en dicho año, la pérdida (y por consiguiente mayor ingreso al FCM) hubiese sido menos de 2 mil millones de pesos.

Finalmente, es necesario precisar que se requieren más estudios, bajos nuevos escenarios, modelos y variables para poder dar mayor sustento a estas recomendaciones, teniendo en cuenta que este tipo de reformas en el país han requerido bastante discusión para su posterior aprobación, como es el caso de la Ley $\mathrm{N}^{\circ} 20.237$.

\section{Referencias}

Bernstein, F. e Inostroza, J. (2009). Modernización municipal y un sistema de evaluación de su gestión. Consorcio para la Reforma del Estado.

Bird, R. M. y Slack, N. E. (1993). Urban public finance in Canada. J. Wiley.

Bravo, J. (2011a). The effects of intergovernmental grants on local revenue: Evidence from chile. IEPUC Documento de Trabajo, (393).

\footnotetext{
${ }^{14}$ No estuvieron disponibles los ingresos del FCM de las comunas Algarrobo, Hualañé y Timaukel para dicho año.

${ }^{15}$ De acuerdo a José Palominos Pino, funcionario de SUBDERE del Departamento de Finanzas Municipales, el valor UTM utilizado en el cálculo corresponde al valor de agosto del año anterior, en este caso agosto del año 2011, \$38.518 pesos.

${ }^{16}$ Fuente: Balance de Ejecución Presupuestario Municipal (BEP) disponible en www.sinim.cl
} 
Bravo, J. (2011b). Fondo común municipal y su desincentivo a la recaudación en chile. Temas de la Agenda Pública, (68).

Cadena, X. (2002). ¿la descentralización empereza? efecto de las transferencias sobre los ingresos tributarios municipales en colombia. Documento CEDE, 8.

CEPAL (1993). Descentralización fiscal: marco conceptual. Serie Política Fiscal No ${ }^{0} 4$.

CEPAL (2005). El sistema municipal y la superación de la pobreza y precariedad urbana en chile. Serie Medio Ambiente y Desarrollo № 111 .

Galilea Ocón, Sergio and Letelier, Leonardo and Ross, Katherine and others (2011). Descentralización de servicios esenciales: los casos de brasil, chile, colombia, costa rica y méxico en salud, educación, residuos, seguridad y fomento.

Henríquez, M., Fuenzalida, J., y Del Fierro, F. (2011). Compensando la desigualdad de ingresos locales: el fondo común municipal (fcm) en chile. Revista Iberoamericana de Estudios Municipales, 2(4):73-104.

Herrera, P. y Francke, P. (2009). Análisis de la eficiencia del gasto municipal y de sus determinantes. Revista Economía, 32(63):113-178.

Horst, B. (2009). Fuentes de financiamiento para gobiernos subnacionales y descentralización fiscal. Consorcio para la Reforma del Estado.

López, G. y Castellanos, A. (2002). Aproximación al concepto de esfuerzo fiscal. Technical report,
Working Paper, 1. Universidad Pompeu Fabra. Centre de Recerca en Economia i Salut (CRES). Barcelona.

Martínez-Costa, C. y Ordeig, O. (2010). Factores determinantes de la eficiencia de las administraciones públicas locales. Dirección y Organización, (42):24-30.

OCDE (2009). Estudios territoriales. Chile. Organización para la Cooperación y el Desarrollo Económico.

Ormeño, H. (2010). Panorama de las finanzas municipales 2001-2008. Instituto Chileno de Municipalidades, Universidad Autónoma de Chile.

Prats, J. (2009). Chile será descentralizado, o no será desarrollado. pp. 11-15.

Raich, U. (2004). Impactos de la descentralización del gasto en los municipios mexicanos. En Guerrero, J. P., editor, Impuestos y gasto público en México desde una perspectiva multidisciplinaria. CIDE-Porrúa.

Rodríguez, J. (1998). The chilean municipal common fund: Conceptual and empirical analysis according to criteria of equity and incentives.

Rosales, M. (2012). Descentralización del Estado y finanzas municipales en América Latina. Flacma.

Valenzuela, J. P. (2008). Análisis descriptivo de la situación financiera de los municipios en chile. Technical report, Inter-American Development Bank. 